\title{
Chemical Peel and Its Implications
}

\author{
Dr Munir Alam \\ MBBS (KE), FRCS (Ire), FRCS (Ed), FCPS (Plastic Surgery) \\ International/European Board of Cosmetic Surgery \\ Associate Professor and HOD Plastic Surgery, Independent Medical College, Faisalabad, Pakistan \\ Consultant Plastic Surgeon, Faisal Hospital, Faisalabad, Pakistan
}

Corresponding author: Dr Munir Alam; mrmuniralam@gmail.com

Received 09 December 2019;

Accepted 17 December 2019;

Published 31 December 2019

Abstract
Background/Objectives: The chemical peels may be used to help ameliorate the scars, to
unsightly pigmentation etc. The treatment modalities used to produce a controlled injury are
(LASER and IPL). Only if the injury is in the proper tissue plane can the desired result
disappointing. If too deep, the results can be catastrophic. Material and Methods: The auth
chemical peeling according to the depth of peeling agent penetration.
\begin{tabular}{|l|l|l|}
\hline Superficial & Medium & Deep \\
\hline TCA $15 \mathrm{gm}$ & TCA $10 \mathrm{gm}$ & TCA $35 \mathrm{gm}$ \\
Croton oil & Mandelic acid $5 \mathrm{gm}$ & Septisol \\
Sesame oil & Salicylic acid $15 \mathrm{gm}$ & Glyserine \\
Distill water & Sesame oil & Croton oil \\
& Croton oil & Distill water \\
\hline
\end{tabular}

\section{PAKPEEL}

This is a prospective study of 3 years from Aug 2016 to Aug 2019. In this study, total number of 216 consecutive chemical peels on 54 patients over a 3 -year period were performed. Age distribution ranges from 20 years to 55 years. Results: The results achieved with these newer chemical formula are highly satisfactory $(80-90 \%)$ for almost all skin conditions treated. Conclusion: In our office setting practice, we perform approximately four sessions of chemical peel weekly and continue skin lightening/spot protection creams and sun protection. This study corroborate evidence superiority of our selected chemical formula.

\section{Keywords: Chemical, Peel, Munir, skin, resurfacing, scars, pigmentation}

\section{Introduction}

A chemical peel is a skin-resurfacing procedure in which a chemical solution is applied to the skin to peel away the accumulation of dead cells and pigment (melanin) on the surface of the skin, enhancing cell renewal and increasing the moisture content of the $\operatorname{skin}^{[1,2]}$. It also helps to stimulate collagen regeneration making skin healthier. The skin can peel or flake off a few days after the peel revealing healthier, smoother and more radiant skin. Recommended home care products help to stimulate skin renewal and sun protection ${ }^{[3,11]}$.

Chemical peels are used to treat fine lines and wrinkles, skin discoloration usually on the face but also on the neck, trunk and hands. A chemical peel can be performed alone or in combination with other cosmetic procedures and results are enhanced if the correct pre-treatment and post-treatment products are used ${ }^{[11]}$.
Chemical peels can be done at different depths from light or superficial to deep depending on desired results (Chart 3). Each peel uses a different chemical solution. Deeper chemical peels produce more-dramatic results and deeper skin rejuvenation but also involve longer recovery times (Picture 2,3) ${ }^{[3,4]}$.

The author has introduced newer formula into the world of chemical peeling according to the depth of peeling agent penetration.

\begin{tabular}{|l|l|l|}
\hline Superficial & Medium & Deep \\
\hline TCA15 gm & TCA10 gm & TCA35 gm \\
Croton oil & Mandelic acid 5 gm & Septisol \\
Sesame oil & Salicylic acid 15 gm & Glyserine \\
Distill water & Sesame oil & Croton oil \\
& Croton oil & Distill water \\
& Distill water & \\
\hline
\end{tabular}


The most common indication includes for superficial PAKPEEL are melasma, post inflammatory hyperpigmentation, superficial scars, freckles and ageing spots. The medium PAKPEEL with its ingredient Salicylic acid indications are mild to severe acne, freckles, sun spots and blemishes. The deep PAKPEEL commonly used for deep hyperpigmentary spots, scars, resistant melasma, chloasma, post-inflammatory hyperpigmentation, freckles, resistant ageing spots.

\section{Material and Methods}

This is a prospective study of three years from Aug 2016 to Aug 2019 and this study was conducted at Faisal Hospital, Faisalabad, Pakistan. The inclusion criteria were clients with aging rhytides ${ }^{[7]}$, pigmentation, acne scarring, ageing spots, premalignant lesions etc. The exclusion criteria includes noncompliant clients, pregnancy \& breast feeding, if the patient has recently waxed, recent surgery, recent use of systemic isotretinoine, recent radiation, herpes virus infection, allergies to chemicals.

As in all cosmetic procedures, a detailed discussion with the patient emphasizing realistic expectation is critical. Patients should be explained that skin will not be "perfect" following a chemical peel (Picture 2). A modest improvement of $50 \%$ to $60 \%$ can be achieved though some may improve more. A careful detailed history should include previous episodes of herpes simplex, prior surgeries in the area, cold intolerance, present and prior medications, pigmentary problems, a history of wound healing following previous surgery and of course realistic expectations, thorough physical examination and relevant investigation performed where indicated.

In this study, total number of two-hundred and sixteen consecutive chemical peels on 54 patients over a 3-year period were performed from Aug 2016 to Aug 2019. Age distribution ranges from 20 years to 55 years with a mean age 29 years $\mathrm{SD} \pm$ 7.4 (chart 1).

There were 42 females and 12 males patients (Chart 2).

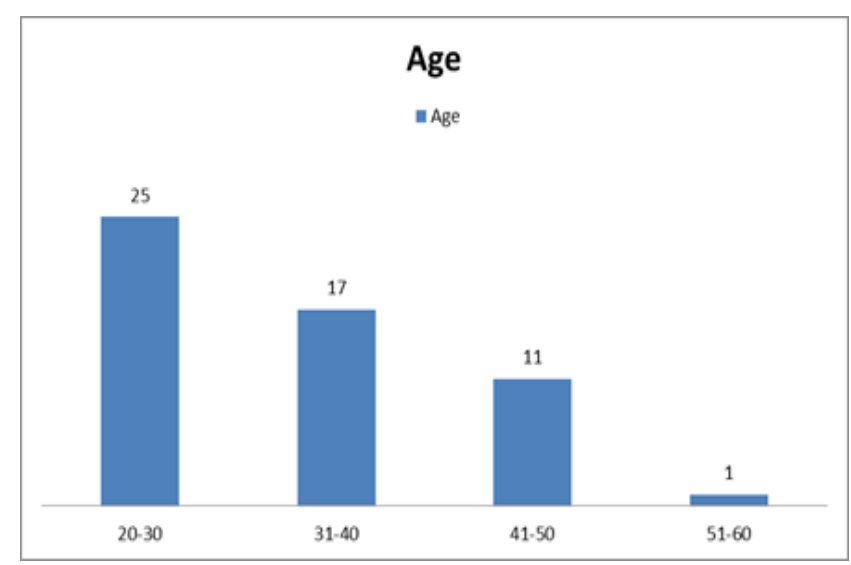

Chart 1: Age Distribution

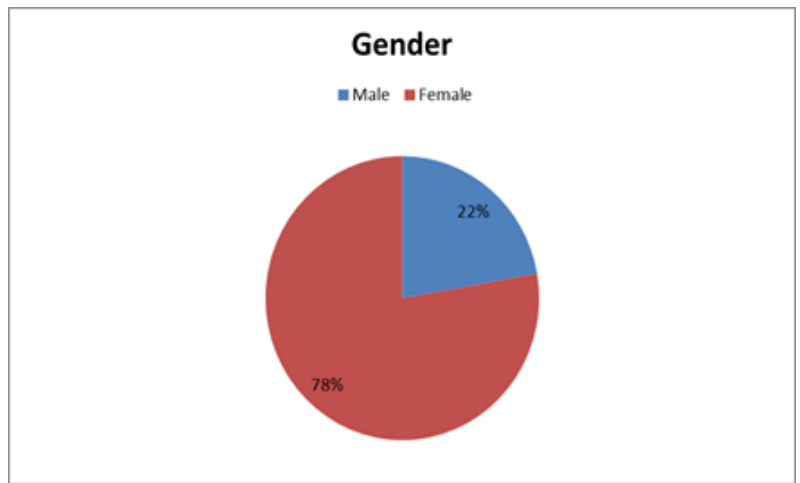

Chart 2: Gender

Forty six patients had successful treatment and eight cases were treated with combined radio frequency (RF) intense pulsed light (IPL) therapy microdermabrasion and chemical peel for acne scarring.

\section{Results}

This is a prospective study in which fifty four patients were treated, out of which 46 patients were treated with various chemical peel treatment and eight patients were treated with chemical peel combined with IPL microdermabrasion for acne scarring. Although statistical analysis show the comparison between these two groups is nonsignificant ( $p$ value 0.106 ), however, clinically satisfactory results achieved in the second group of patients.

In our office setting practice, the author perform approximately four sessions of chemical peel weekly and continue skin lightening/spot protection creams and sun protection. The results achieved with these new innovative PAKPEEL are highly satisfactory $(80-100 \%)$ for almost all skin conditions treated by using visual analogue scoring system.

\section{Discussion}

A new patented technology allows Easy Trichloracetic Acid (TCA) Controls pain to safely give results comparable to a papillary dermis depth, with a very discreet burning sensation only. Efficacy has increased especially on pigment problems. As the skin is dynamic structure, the session of peeling may require a repeat session almost every six months or yearly to maintain the desired results $^{[2,4,6]}$.

In comparison to PAKPEEL, the Skin Tech brand is the most famous and internationally known medium depth chemical peel for skin regeneration, considered as one of the safest, the most effective and the easiest peeling. The Easy TCA is used to treat facial fine lines, photo-ageing, acne problems, acne scars, pigment problems (hyperpigmentation), melasma, chloasma, keratosis of hands, forearm and body stretch marks, "ageing spots", etc ${ }^{[5]}$

Chart 3: Depth of Penetration

\begin{tabular}{|c|c|c|c|c|}
\hline Agent & Superficial Epidermis & Deep Epidermis & Papillary Dermis & Reticular Dermis \\
\hline 1. Superficial & & & & \\
\hline 2. Medium & & & & \\
\hline 3. Deep & & & & \\
\hline 4.Combination of IPL Microdermabrasion & & & & \\
\hline
\end{tabular}


Among the superficial chemical peels, the glycolic acid is a natural constituent of cane sugar and the smallest molecule of the alphahydroxy (AHA) family.

In practice, Combined use of Glycolic and Lactic acids to exfoliate surface cells and hydrate the skin. This peel revitalises the appearance of photodamaged skin.

Skin ceuticals gel peel SM are formulated to address pore congestion, wrinkles, other signs of ageing. This peel combines Salicylic and Mandelic acids to manage problematic skin and promote exfoliation.

Obagi Radiance Peel is a gentle chemical peel for a healthy, bright complexion with a blend of $20 \%$ Salicylic Acid, Lactic and Glycolic Acid. Nevertheless, chemical peels may result in drastic complication such as toxic shock syndrome ${ }^{[8]}$.

Our PAKPEEL Superficial peel contain low dose trichloroacetic acid (15\%) which is a soft peeling continuous and non invasive cutaneous renewal and is indicated for melasma/chloasma (Picture 1,2), post inflammatory hyper pigmentation, superficial scars, freckles, age spots etc. It is useful on dyschromia, photoaging, seborrheic keratosis, increases tonicity and elasticity, increases brightness, and is by far safer and less aggressive

Medium PAKPEEL contains salicylic acid which is antiseptic and keratolytic help ameliorate the acne scarring. Mandelic acid is non-comedogenic, anti-microbial and soothing effect and is smoothing to skin restoration. Mandelic acid is epidermal reactivator, moisturizer with a firming effect. Trichloroacetic acid (TCA) has exfoliating and antibacterial action.

Acne treatment with medium PAKPEEL is a highly advance therapy for acne. It has been introduced recently, but has done wonders for the acne issue. It is the most effective treatment ever invented for acne problems and kicks out acne from your skin quicker than any other traditional treatments! In this process, each pimple of acne is treated individually for the best and enduring results.

\section{Picture 1:}

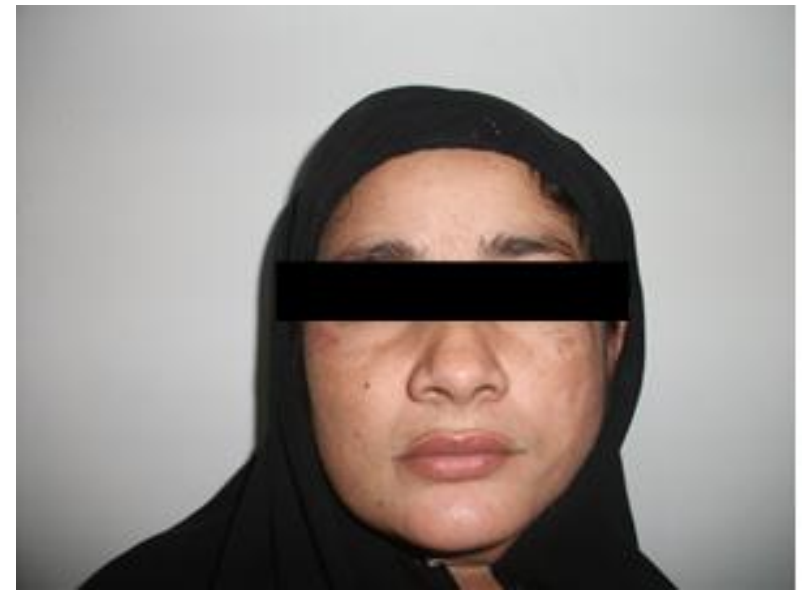

A: Before Peeling

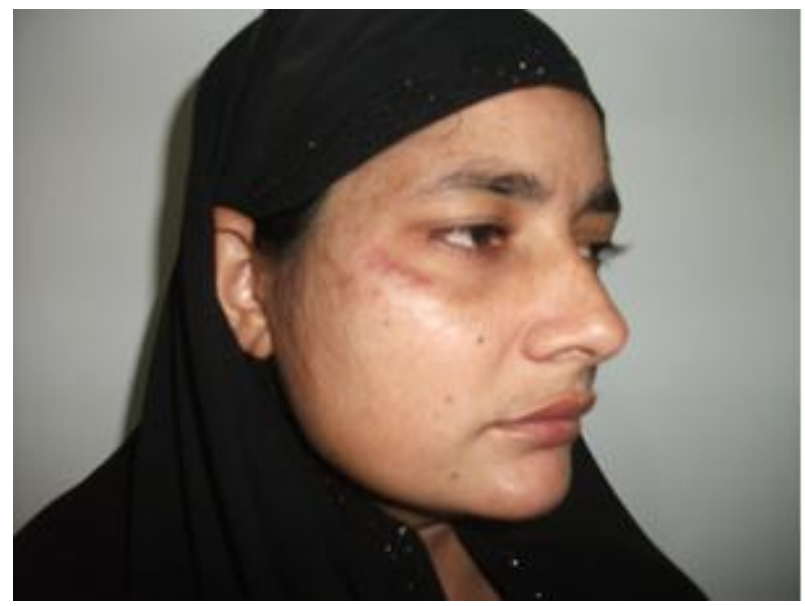

C: Before Peeling

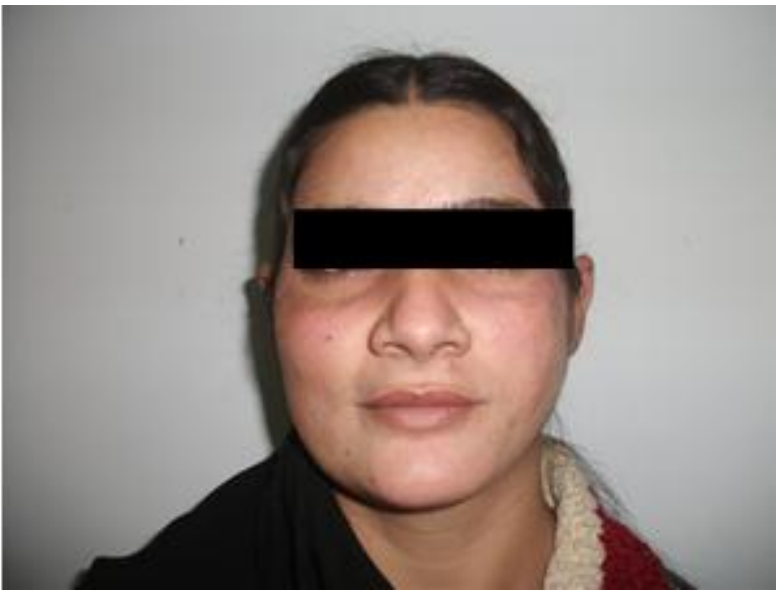

B: 3 Months after Peeling

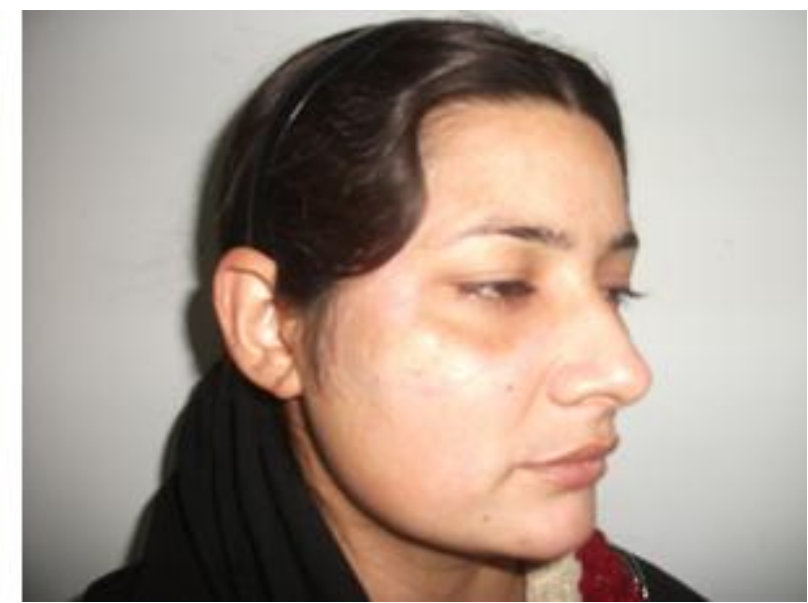

D: 3 Months after Peeling 


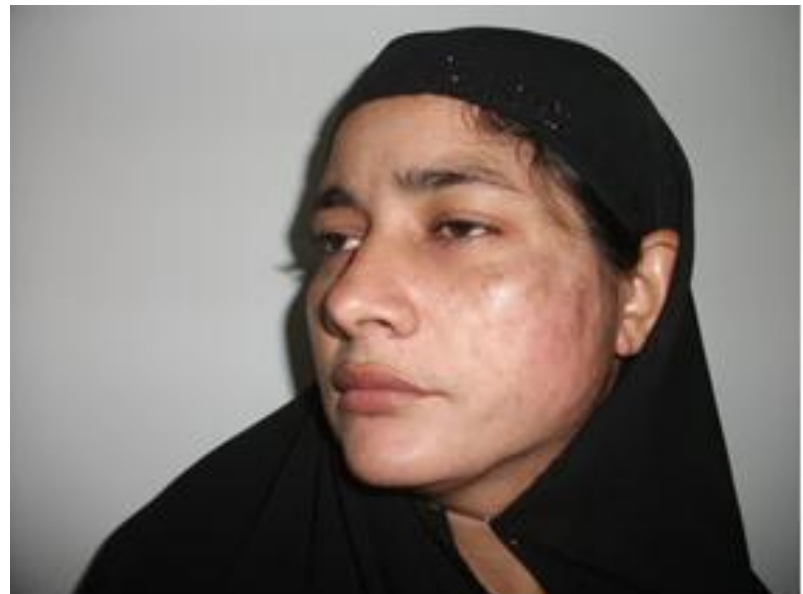

E: Before Peeling

Deep peel contain high concentration of TCA (35\%) is a intensive peeling, with a whitening and hypopigmenting action. This is recommended for skin with visibly dilated pores. It is antibacterial, keratolytic action. It has whitening and brightening effect. Sesame oil exfoliate the skin, enhance collagen and elastin synthesis/remodelling.

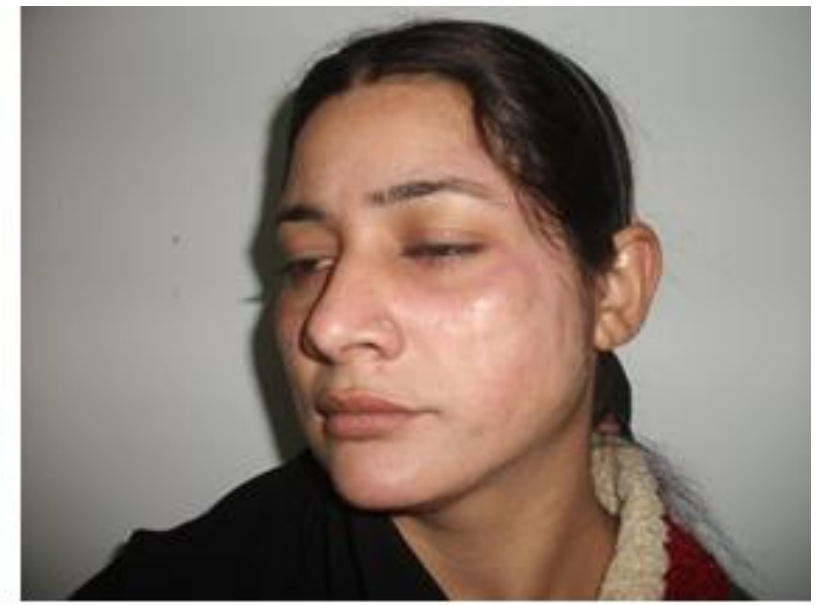

F: 3 Months after Peeling

Deep peeling guarantees a strong and long-lasting cutaneous renewal with a lifting effect. This is highly indicated for thick skin, resistant melasma and/or skin with deep hyperpigmented postburns cars (Picture 3).

\section{Picture 2:}

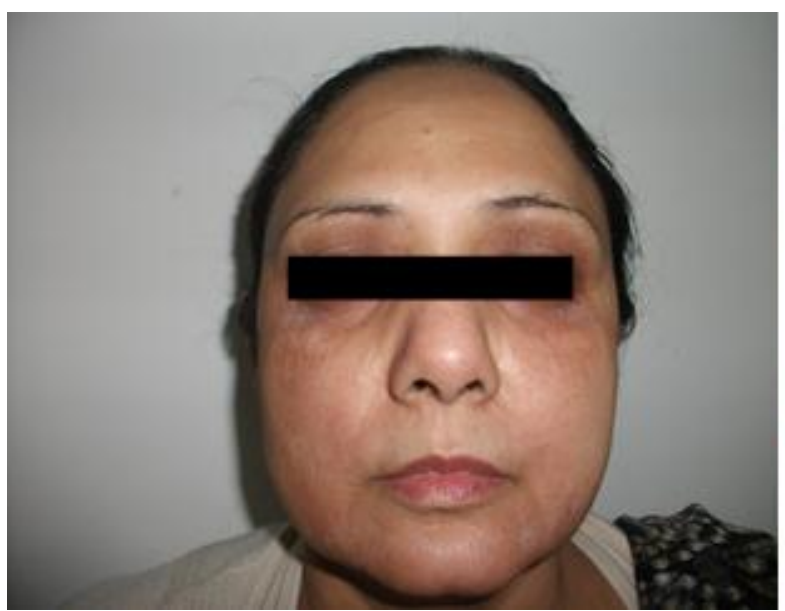

A: Before Peeling

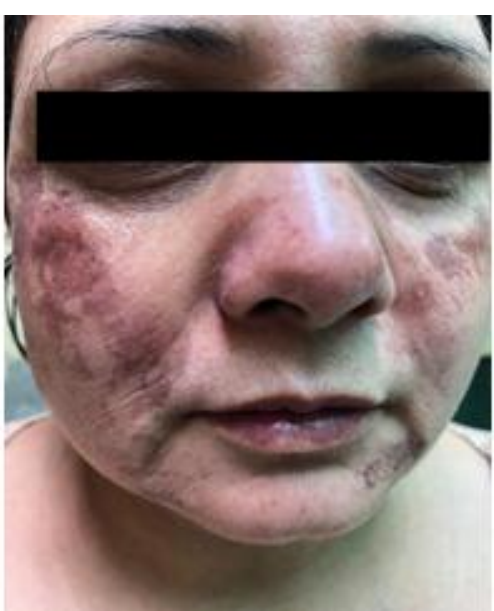

B: 3 - 4 weeks after Peeling

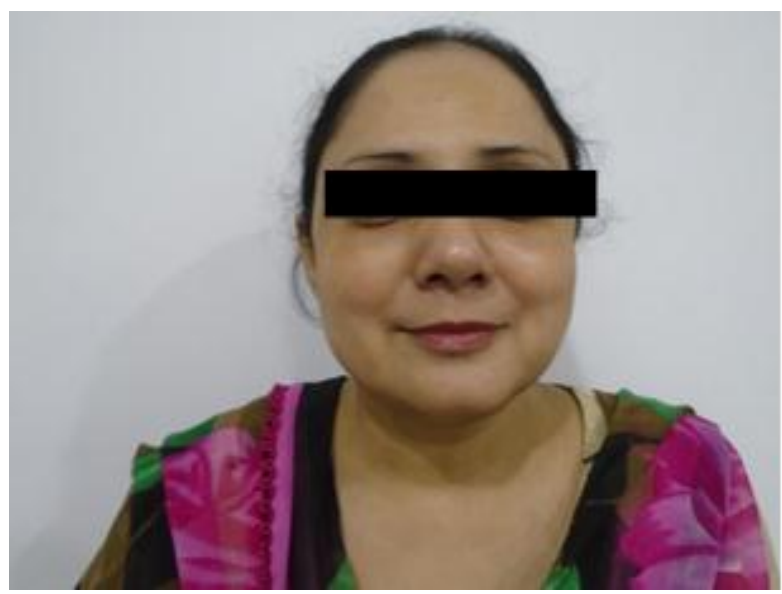

C: 5 Months after Peeling

In my experience, we have not encountered with problems such as wound infection, this risk may be reduced due to appropriate postpeel wound care. Pigmentary problems are common especially hyperpigmentation. Hyperpigmentation is inflammatory in nature. It is possible as a result of melanocyte overstimulation secondary to trauma and can develop from acne, chronic dermatitis eruptions, and trauma to the skin by the use of chemical peels. Patients at risk for hyperpigmentation should be treated aggressively both before and after chemical peeling with agents such as retinoic acid, glycolic acid, hydroquinone, kojic acid, or other similar compounds. If the hyperpigmentation is deep in the dermis, conservative treatment with the listed agents and "tincture of time" proven the most prudent. 


\section{Picture 3:}

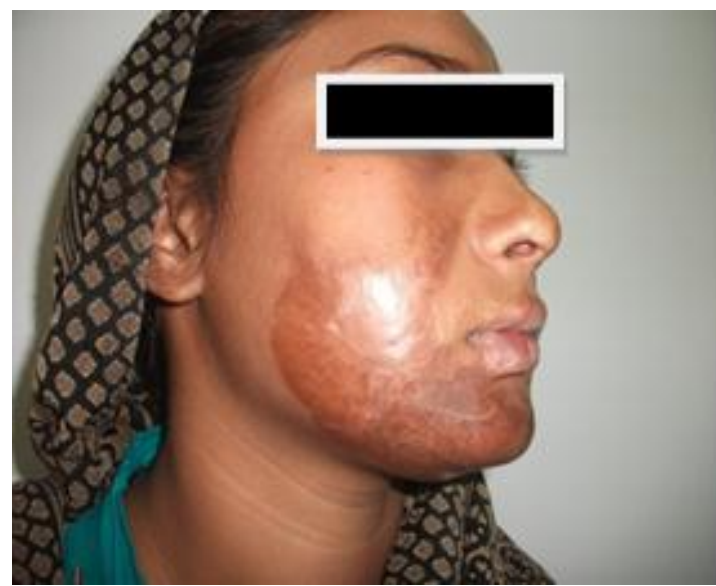

A: Before Peeling

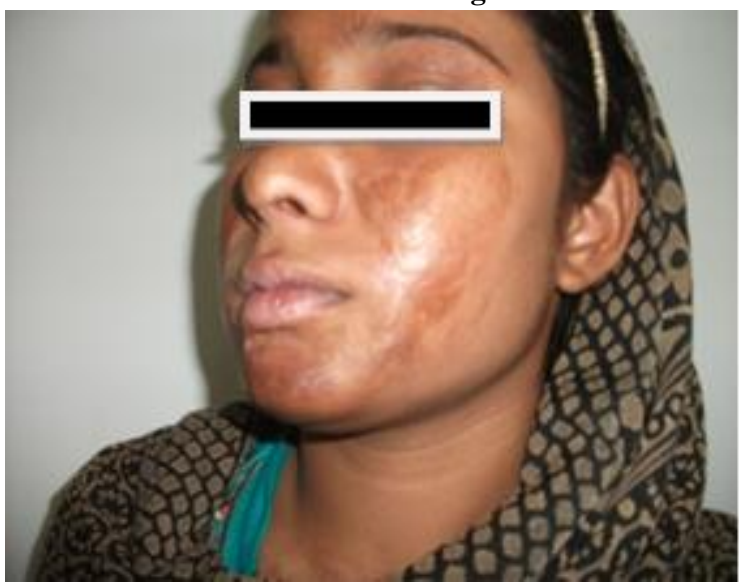

C: Before Peeling

Perhaps the most dreaded complication of chemical peeling is scarring. The deeper the peel the higher the risk of scarring. To avoid these complication due to chemical peeling, I prefer to perform four sessions of peeling weekly taking into consideration the response of skin to chemicals and achieving the desired results ${ }^{[12]}$. However, the deep peeling may result in resurfacing upto the deeper dermal layer. Only the tincture of time and soothing/moisturising lotion and sun protection is necessary to achieve the desired results after the episode of acid burn (Picture 2).

Acne scarring is caused by the body effectively putting "too much effort" into healing the wounds caused by acne, meaning that there is an excess of collagen built up in the dermis. This collagen may stay in place permanently creating acne scars. Traditional dermabrasion have been combined with other techniques such as punch grafting of deeper, wider, ice picks scars trephined using various-sized punches. The tissue is discarded and replaced with similarly sized punch grafts from normal skin and subsequently dermabraded. The results achieved using these techniques are unpredictable ${ }^{[9]}$.

Dermabrasion was first introduced by Dr Abner Kurtin in 1953, a New York dermatologist ${ }^{[13]}$. This traditional technique combines a hand engine with either diamond-studded fraises or wire brushes. It is indicated for scarring especially the acne scarring or photodamage skin. Dermabrasion is extremely technique dependent. The art of dermabrasion is knowing how deep to go. If one goes too deeply, scarring will be created, If one does not go deeply enough, the results will be suboptimal. However, these complications are most likely encountered with the use of traditional dermabrader, we have achieved highly

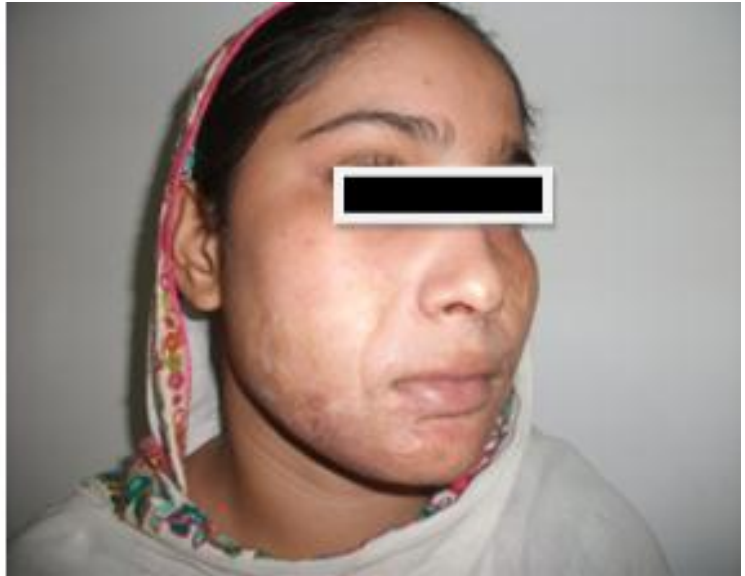

B: 4 Months after peeling

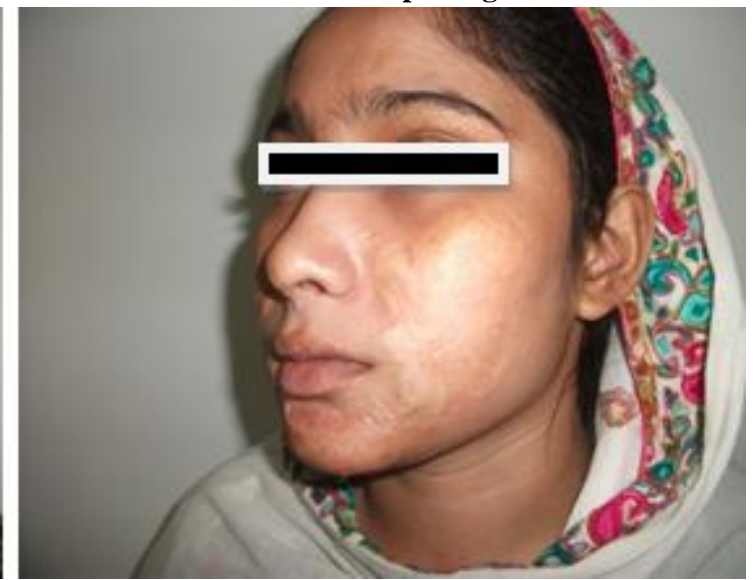

D: 4 months after peeling

satisfactory results with the most modern technique of RF IPL combined with newer PAKPEELs.

Complications are also more likely with darker skin types, certain peeling agents, and sun exposure. They can range from minor irritations, uneven pigmentation to permanent scarring. In very rare cases, complications can be life-threatening ${ }^{[14,15,16,17,18]}$.

To combat this difficult situation, we have been able to use with success, combination of RF Intense Pulse Light therapy with microdermabrasion and 35\% TCA in a controlled manner with excellent results.

\section{Conclusion}

The art of chemical peel require a well trained and experienced person. The patient should be informed about possible post peel expectation and the time frame to achieve the desired results. Although postpeel wound care responsibility will lie with the patient, the direction comes from the treating doctor. It is important to remember that the skin has been wounded and is in a vulnerable state. Like any wound healing, it requires careful wound treatment and camouflage with temporary make ups for upto three months post peel recuperative phase. As the skin is a dynamic structure, the session of peeling may require every six months or yearly to maintain the desired results.

\section{Abbreviations}

Light amplification of stimulated emission of radiation (LASER), Intense pulse light therapy (IPL), Trichloroacetic acid (TCA). 


\section{Conflicts of Interest}

None

\section{Funding Statement}

No financial interest

\section{Acknowledgments}

DERMASCIENCE pharmaceutical company, Lahore, Pakistan. Manufacturer and sole distributor of PAKPEEL.

\section{References}

[1] Baker T J and Gordon H I, The ablation of rhytides by chemical means: A preliminary report J. Fla. Med Assoc. 48: 451, 1961.

[2] Paul S. Collins. A, The chemical peel, Clinics in Dermatology, Vol 5 (4) Oct-Dec 1987, 57-74.

[3] Eileen $\mathrm{C}$ and Lawrence S, Superficial and medium-depth chemical peels, Clinics in Dermatology Vol 26 (2) MarApril 2008,209-218.

[4] Lynn A D, Scott M Det al; Guidelines of care for chemical peeling, Journal of the American Academy of Dermatology, Vol 33 (3), Sep 1995, 497-503.

[5] Francine L. V and Scott R. B, Histologic study of dermabrasion and chemical peel in an animal model after pretreatment with Retin-A, Aesthetic Plastic Surgery, May 1995 Vol 19 (3), 243-246.

[6] Dinner MI and Artz JS, The art of the trichloroacetic acid chemical peel, Clinics in Plastic Surgery Jan 1998, Vol 25(1):53-62
[7] Thomas J B, Chemical Face Peeling and Rhytidectomy: A combined approach for facial rejuvenation, Plastic \& Reconstructive Surgery: Feb 1962 - Vol 29 (2) 199-207

[8] LoVerme, William E. Drapkin, Mark S et al; Plastic \& Reconstructive Surgery: July 1987 Vol 80(1)115-118.

[9] Grabb and Smith's Plastic Surgery, 5th Edition pp605, Philadelphia 1997.

[10] Phillip R L, Mark M, Robert Y, Comparison of the Laser and Phenol Chemical Peel in Facial Skin Resurfacing, Arch Otolaryngol Head Neck Surg. 2000;126(10):11951199.

[11] Brian P M, Brad M, Gary M, Gaylon M, The Etiology of Prolonged Erythema after Chemical Peel, Dermatologic Surgery, Volume 24, Issue 3, Mar 1998,337-341.

[12] Alam M, Iqbal MJ, Akhtar R. Chemical Peels: What to Treat and Expect?. J Dermatol Plast Surg. 2017; 2(2): 1013.

[13] Robbins N, Dr. Abner Kurtin, Father of Ambulatory Dermabrasion, The Journal of Dermatologic Surgery and Oncology, Volume14, Issue4, April 1988, Pages 425431.

[14] Nanma Nikalji, Kiran Godse, Jagdish Sakhiya et al; Complications of Medium Depth and Deep Chemical Peels; J Cutan Aesthet Surg. 2012 Oct-Dec; 5(4): 254 260. doi: 10.4103/0974-2077.104913

[15] Brody HJ. Complications of chemical peeling. J Dermatol Surg Oncol. 1989;15:1010-9.

[16] Anitha B. Prevention of complications in chemical peeling. J Cutan Aesthet Surg. 2010;3:186-8.

[17] Spira M, Gerow FJ, Hardy SB. Complications of chemical face peeling. Plast Reconstr Surg. 1974;54:397-403.

[18] Khunger N, Vedamurty M, Arsiwala S. Step by Step Chemical Peels. 1st ed. New Delhi: Jaypee Medical Publishers; 2009. Complications; pp. 279-97. 